\title{
PREVALENCIA Y FACTORES ASOCIADOS A SINTOMATOLOGÍA DEPRESIVA EN ESTUDIANTES DE MEDICINA DE LA PROVINCIA DE ICA, PERÚ
}

\author{
Martha Legua-Flores ${ }^{1, a}$, C. Hugo Arroyo-Hernández ${ }^{2, b}$
}

1. Facultad de Medicina. Universidad Nacional San Luis Gonzaga. Ica. Perú.

a. Magíster en Salud Pública. b. Médico Cirujano

\begin{abstract}
RESUMEN
Objetivo: Determinar la prevalencia y factores asociados a sintomatología depresiva en estudiantes de medicina de la provincia de Ica, Perú. Material y método: Estudio de tipo transversal analítico realizado en julio del 2007, en estudiantes de medicina de la Universidad Nacional de Ica; se aplicó de forma autoadministrada, voluntaria y anónima el test de depresión de Zung, indagando además factores protectores. Se uso estadística descriptiva y análisis de regresión logística. Resultados: Un 22\% de estudiantes presento algún grado de sintomatología depresiva, siendo en el $2 \%$ una depresión de forma severa; Entre los factores asociados a depresión se encontró que eran significativos para: convivencia solo o con algún familiar ( $p=0,027$; OR=2,07; IC95\% 1,08-3,94), lugar de residencia de sus padres en otro lugar ( $p=0,034 ;$ OR=1,99; IC95\% 1,05-3,77), tiempo para tareas académicas nunca o casi nunca ( $p=0,001$; OR=7,15; IC95\% 2,97-7,94). Conclusiones: Existe una alta prevalencia de estudiantes de medicina con algún grado de depresión en el grupo sin soporte familiar protector y distanciamiento de los vínculos parentales. ( Rev. méd. panacea 2011; 1:28-33).
\end{abstract}

Palabras clave: Depresión; Estudiantes de Medicina; Perú.

\section{PREVALENCE AND RELATED FACTORS WITH DEPRESSIVE SYMPTOMS IN MEDICAL STUDENTS FROM ICA PROVINCE, PERU.}

\begin{abstract}
Objetive: To determine the prevalence and related factors with depressive symptoms in medical students from Ica, Peru. Material and methods: Transversal-analytical study realized in July 2007, in students of medicine from Ica in a National University; the depression test of Zung was autoapplied, voluntary and anonymously, also searching for protecting factors. It was used descriptive statistics and logistic- regression analysis. Results: $22 \%$ of students presented some depression grade, being $2 \%$ of the severe form, in the related factors associated to depression it was found that these were significant to: living alone or with some relative $(p=0,027 ;$ OR $=2,07 ; 95 \%$ IC $[1,08-3,94])$; the parents place of residence is someplace else $(p=0,034 ;$ OR $=1,99 ; 95 \%$ IC $[1,05-3,77])$; time for academic tasks never or hardly ever $(p=0,001 ;$ OR=7,15; 95\% IC $[2,97-$ $7,94]$ ). Conclusions: There is a high prevalence of medical students with some depression grade in the group without the protecting familiar support and separation of the parental links. (Rev. méd. panacea 2011; 1:28-33).
\end{abstract}

Key words: Depression; Medical Students; Peru.

\section{INTRODUCCIÓN}

Depresión es un trastorno psiquiátrico con un curso crónico y recidivante, que afecta principalmente a adolescentes el que es considerado como el grupo de mayor riesgo; entre otros grupos de riesgo tenemos, personas con: antecedentes de trastornos afectivo familiar, experiencias de muerte parental temprana, no existiendo correlación significativa con los estratos económicos ${ }^{(1,2)}$.

La ausencia de salud mental en las personas impide, superar obstáculos cotidianos y avanzar con su proyecto de vida, dando lugar a falta de productividad tanto individual como social ${ }^{(3)}$. Según la Organización Mundial de la Salud, la depresión de tipo intensa o severa es la principal causa de discapacidad y la cuarta de las diez principales causas en la carga de morbilidad a nivel mundial ${ }^{(4)}$.
En Perú, estudios realizados por el Instituto Especializado de Salud Mental, Honorio Delgado-Hideyo Noguchi, muestran que la prevalencia de depresión en personas que habitan en Lima metropolitana es de 18,2\%; en la Sierra $16,2 \%$ y en la Selva $21,4 \%$; dando como resultado que en un mes $1 \%$ de personas en Lima Metropolitana, $0,7 \%$ en la Sierra y $0,6 \%$ en la Selva, presenten intentos suicidas ${ }^{(5-7)}$.

Diversos autores en todo el mundo han analizado la salud mental de los estudiantes de medicina, quienes se encuentran expuestos a altos niveles de estrés, situación que puede repercutir en su rendimiento intelectual, salud física y bienestar psicosocial; demandando mayor esfuerzo de adaptación del estudiante, en especial durante los primeros años de estudio ${ }^{(8,10-12)}$. Considerando además la falta de tiempo para realizar actividades recreativas, esta puede ejercer en ellos una perjudicial carga de estrés ${ }^{(9)}$. 
El soporte social definido por Cobb, como "la información que conduce a creer que uno es cuidado, amado y estimado" es identificado como un factor protector, que beneficia la salud y favorece la recuperación en caso de enfermedad; dentro de este contexto, el ambiente familiar, la calidez parental y calidad del vínculo, promueven el funcionamiento adaptativo en adolescentes y estudiantes de medicina expuestos a marcado estrés ${ }^{(13-15)}$.

La Facultad de Medicina de la Universidad Nacional San Luis Gonzaga de Ica, ubicada en la provincia de Ica, a 300 km al sur de la ciudad de Lima, en la costa del Perú; recibe anualmente, luego de un riguroso proceso de selección, un promedio de 60 jóvenes y adolescentes, gran número de ellos provenientes de otras provincias, quienes durante el transcurso de la carrera residen en habitaciones alquiladas, aledañas a la Facultad de Medicina o con algún familiar que los alberga en un primer momento.

El objetivo de nuestra investigación fue determinar la prevalencia y factores asociados a sintomatología depresiva en estudiantes de medicina de la provincia de Ica,

\section{MATERIAL Y MÉTODOS}

Estudio de tipo observacional, transversal y analítico, realizado durante el mes de julio del 2007. Los participantes incluidos en la investigación fueron 227 estudiantes de pregrado del primer a sexto año de estudios de la Facultad de Medicina de la Universidad Nacional San Luis Gonzaga de Ica, matriculados en el año académico 2007, escogidos mediante una muestra no probabilística por conveniencia, estratificada por año de estudios de una población total de 458 estudiantes.

Se uso como instrumento de recolección de datos una encuesta estructurada, autoadministrada, voluntaria y anónima; constituida en dos partes, la primera que recogía información sobre características sociodemográficas como: edad, sexo, año de estudios, tipo de convivencia, lugar de residencia de los padres, tiempo para cumplir con tareas académicas, tiempo para la recreación; la segunda parte incluía, el Test de Depresión de Zung (Self-Rating Depression Scale, SDS), validado en Perú por Novara y col (16). El test de Zung explora síntomas de episodios depresivos recientes y consta de 20 preguntas, cada una de ellas con 4 alternativas tipo Likert, donde la suma de la puntuación total de preguntas permite identificar la severidad o nivel de depresión en la persona; así tenemos que: <49 puntos es considerada normal o sin depresión, 50-59 puntos depresión leve, 60-69 puntos depresión moderada $\mathrm{y} \geq 70$ puntos depresión intensa o severa.
Tabla 1. Características sociodemográficas de la población estudiada.

\begin{tabular}{lcc}
\hline \multicolumn{1}{c}{ Variables } & $\mathbf{n}=\mathbf{2 2 7}$ & $\mathbf{( \% )}$ \\
\hline $\begin{array}{l}\text { Edad } \\
\text { Sexo }\end{array}$ & $22 \pm 3^{*}$ & $(17-39) \dagger$ \\
$\quad$ Masculino & 132 & $(58,1)$ \\
$\quad$ Femenino & 95 & $(41,9)$ \\
Año de estudio & & \\
$\quad$ Primero & 38 & $(16,7)$ \\
$\quad$ Segundo & 38 & $(16,7)$ \\
$\quad$ Tercero & 37 & $(16,3)$ \\
$\quad$ Cuarto & 40 & $(17,6)$ \\
$\quad$ Quinto & 38 & $(16,7)$ \\
$\quad$ Sexto & 36 & $(15,9)$ \\
Convivencia & & \\
$\quad$ Con padres & 118 & $(52,2)$ \\
$\quad$ Solo o con algún familiar & 108 & $(47,8)$ \\
Residencia de padres & & \\
$\quad$ Ica & 147 & $(64,8)$ \\
$\quad$ Otro lugar & 80 & $(35,2)$ \\
Tiempo para tareas académicas & & \\
$\quad$ Siempre o casi siempre & 202 & $(89,0)$ \\
$\quad$ Nunca o casi nunca & 25 & $(11,0)$ \\
Tiempo para recreación & & \\
$\quad$ Siempre o casi siempre & 131 & $(57,7)$ \\
$\quad$ Nunca o Casi nunca & 96 & $(42,3)$ \\
\hline
\end{tabular}

* Media \pm desviación estándar; † (mínimo - máximo)

La encuesta fue realizada en la oficina de tutoría y bienestar estudiantil de la Facultad de Medicina, dentro del periodo de visitas para la realización de exámenes médicos a estudiantes; siendo administrada con la supervisión y coordinación del director de dicha oficina y los autores.

Luego de pasar por un control de calidad, los registros de las encuestas fueron ingresados a una base de datos, creada en el software SPSS v.15.0; los resultados del Test de Zung se categorizaron en dos variables: estudiantes con ningún grado de depresión si el resultado era normal y, estudiantes con algún grado de depresión si el resultado era leve, moderada o intensa. Para el análisis de los datos se uso estadística descriptiva análisis bivariado y análisis de regresión logística, para ello se uso el software Stata 9 (Stata Corporation, College Station, TX). Se determino la prevalencia de depresión y sus intervalos de confianza al $95 \%$, se considero $p<0,05$ como valor de significación estadística; se uso el Odds Ratio (OR) -con sus Intervalos de Confianza al 95\% (IC 95\%)- para determinar los niveles 
de asociación con los factores de riesgo para presentar algún grado de depresión.

Tabla 2. Prevalencia de sintomatología depresiva en estudiantes de medicina de la provincia de Ica.

\begin{tabular}{lcc}
\hline \multicolumn{1}{c}{ Variables } & n/N & Prevalencia \\
\hline Global & $50 / 227$ & 22,0 \\
Edad & $21 \pm 3^{*}$ & $(17-30) \dagger$ \\
Severidad depresiva & & \\
$\quad$ Leve & $36 / 227$ & 15,9 \\
$\quad$ Moderada & $9 / 227$ & 3,9 \\
$\quad$ Intensa & $5 / 227$ & 2,2 \\
Sexo & & \\
$\quad$ Masculino & $24 / 132$ & 18,2 \\
$\quad$ Femenino & $26 / 95$ & 27,4 \\
Año de estudios & & \\
$\quad$ Primero a tercero & $30 / 113$ & 26,5 \\
$\quad$ Cuarto a sexto & $20 / 114$ & 17,5 \\
Convivencia & & \\
$\quad$ Con Padres & $19 / 118$ & 16,1 \\
$\quad$ Solo o con algún familiar & $31 / 108$ & 28,7 \\
Residencia de padres & & \\
$\quad$ Ica & & \\
$\quad$ Otro lugar & $26 / 147$ & 17,7 \\
Tiempo para tareas académicas & & 30,0 \\
$\quad$ Siempre o casi siempre & $35 / 202$ & 17,3 \\
$\quad$ Nunca o casi nunca & $15 / 25$ & 60,0 \\
Tiempo para recreación & & \\
$\quad$ Siempre o casi siempre & $24 / 131$ & 18,3 \\
$\quad$ Nunca o casi nunca & $26 / 96$ & 27,0 \\
\hline Meda; desvacion estándar & &
\end{tabular}

* Media; \pm desviación estándar; † (mínimo - máximo)

\section{RESULTADOS}

Se incluyeron un total de 227 estudiantes de medicina, cuya edad media fue de 223 años, edad mínima 17 y máxima 30 ; de los cuales el $58,1 \%$ son del sexo masculino. La distribución por año de estudios fue homogénea en todos los años.

Con respecto a las preguntas: con quien vive en lca $52,2 \%$ manifestó vivir con sus padres y $47,8 \%$ solos o con algún familiar, el lugar de residencia de sus padres fue $64,8 \%$ en Ica y $35,2 \%$ en otro lugar. El $89 \%$ de estudiantes refirió tener tiempo siempre o casi siempre para cumplir con las tareas académicas, el 42,3\% manifestó no disponer de tiempo para su recreación (Tabla 1).

La prevalencia global de estudiantes de medicina que presentaron algún grado de depresión fue del $22 \%$, afectando principalmente aquellos con edad media de 213 años. Según la severidad depresiva encontrada el 19,8\% fue leve, 3,9\% moderada y $2,2 \%$ intensa; la prevalencia de estudiantes que presentaron algún grado de depresión fue mayor para el sexo femenino $(27,4 \%)$, en general afecto más en los tres primeros años de estudios (26,5\%). Otros factores asociados fueron: vivir solo o con algún familiar $(28,7 \%)$, lugar de residencia de sus padres en otro lugar $(30 \%)$, tiempo para cumplir con tareas académicas nunca o casi nunca $(60 \%)$ y, tiempo para la recreación, nunca o casi nunca $(27 \%)$ (Tabla 2$)$.

En el análisis bivariado se encontró como factores asociados para presentar algún grado de depresión: vivir solo o con algún familiar (OR=2,07; IC 95\% [1,08-3,94] $p=0,027)$, el lugar de residencia de sus padres en otro lugar (OR=1,99; IC 95\% $[1,05-3,77] p=0,034)$; y tener escaso tiempo para las tareas académicas $(O R=7,15$; IC $95 \%$ [2,97 - 7,94] $p=0,001)$; no se encontró asociación estadísticamente significativa con las variables sexo, año de estudios y tiempo para la recreación (Tabla 3).

Los factores asociados con algún grado de depresión en el modelo de regresión logística fueron: vivir solo o con algún familiar y tener tiempo para las tareas académicas nunca o casi nunca (Tabla 4).

\section{DISCUSIÓN}

Nuestros resultados muestran una prevalencia global de sintomatología depresiva superior al de la población general $(5,6,7)$, y al encontrado en estudiantes de medicina de una universidad pública de otra provincia peruana; sin embargo, son inferiores a los encontrados en una universidad pública y dos universidades privadas en la Ciudad de Lima (Tabla 5) $(8,20,21,26)$.

La relación de estudiantes con presencia de algún grado de depresión es uno de cada cuatro, ubicándose en una escala intermedia en comparación con los resultados encontrados por Perales y Col. en dos universidades públicas peruanas ${ }^{(8)}$.

La mayor prevalencia para presentar sintomatología depresiva, en estudiantes de medicina, del sexo femenino podría está relacionada a una diferencia de patrones de respuesta frente al estrés, con respecto a estudiantes de sexo masculino ${ }^{(17,22)}$; así mismo, la diferencia existente entre la mayor prevalencia de depresión en los primeros años de estudios que disminuye durante el transcurso de los últimos años podría indicar una disminución de la ansiedad asociada al tiempo de permanencia y la capacidad de adaptación progresiva a la carga académica que experimenta el alumno ${ }^{(23,24)}$. 
Tabla 4. Factores independientes asociados a sintomatología depresiva en estudiantes de medicina de la provincia de lca. Modelo de regresión logística

\begin{tabular}{lcccc}
\hline \multicolumn{1}{c}{ Variables en el modelo } & OR & (IC 95\%) & p \\
\hline Vivir solo o con algún familiar & 2,3 & $(1,13-4,71)$ & 0,01 \\
Residencia de padres en otro lugar & 2,01 & $(0,97-4,20)$ & 0,06 \\
Tiempo para tareas académicas nunca o casi nunca & 7,07 & $(2,68-19,49)$ & 0,0001 \\
\hline
\end{tabular}

En nuestro estudio encontramos un alto porcentaje de estudiantes que viven solos o con algún familiar, asegurando un distanciamiento de vínculos con el núcleo familiar, lo que se considera además como una causa de depresión en universitarios, debido al carecimiento del apoyo afectivo y emocional que brindan los padres; evidenciándose una mayor prevalencia de depresión dentro de este grupo. Esto avala que el soporte social brindado por los padres es un factor protector ${ }^{(15,19)}$. Esta característica no se halla incluida en otras investigaciones publicadas, sobre depresión en estudiantes de medicina en Perú, de donde se rescatan otros factores protectores como son tener una relación de pareja y la religión ${ }^{(8)}$.

En una investigación realizada en estudiantes de pregrado de una universidad colombiana, se encontró un mayor porcentaje de depresión en estudiantes que viven en apartamentos compartidos con otros compañeros; aquí se señala que el compartir un espacio físico no garantiza necesariamente una buena calidad de: interacción, sensación de pertenencia, seguridad e intimidad ${ }^{(18)}$.

Las características de la población estudiada muestra que un gran porcentaje refiere tener tiempo necesario para cumplir con sus tareas académicas; sin embargo, encontramos una asociación significativa en estudiantes con algún grado de depresión y poco tiempo para cumplir las tareas académicas. Cabe mencionar que algunos estudiantes dedican tiempo para trabajar, con el objetivo de solventar y continuar sus estudios en la universidad o contribuir con el sustento económico de su hogar; estos son factores estresantes por si mismos los que pueden llevar a presentar síntomas ansioso depresivos, convirtiéndose en una causa extra.

Por otro lado, la disminución del interés por la realización de tareas académicas como consecuencia de la presentación de algún grado de depresión, podría ser interpretada por los estudiantes como la falta de tiempo para realizar tareas académicas, dando lugar a dificultades en el rendimiento académico $^{(12)}$.
A pesar de no encontrarse como factor asociado para presentar algún grado de depresión, uno de cada dos estudiantes manifestó la ausencia de tiempo destinado a la recreación, siendo esto mayor a lo hallado por Perales y col, en dos universidades públicas peruanas ${ }^{(8)}$; es probable que esta característica guarde alguna relación con el hecho de vivir solo o con algún familiar.

Muchos estudiantes de medicina, que se alejan de sus hogares, dejan sus lugares de origen en busca de una preparación universitaria enfrentándose a nuevas situaciones y ambientes estresantes, que podrían generar en ellos un deterioro de su salud mental. Esto dependería del grado de adaptación logrado y del hallazgo de un necesario soporte social, lo que nos permite manifestar la necesidad de una mayor atención en el cuidado de la salud mental de este grupo de estudiantes de medicina.

Si bien existe creciente interés en las investigaciones sobre depresión en estudiantes de medicina, es importante empezar a buscar acciones para salvaguardar su bienestar mental y social, implementando programas de vigilancia que involucren a las Facultades de Medicina en su prevención, diagnóstico, manejo efectivo y evaluación de sus intervenciones ${ }^{(25)}$.

Consideramos que los futuros profesionales de la salud deben tener una formación capaz de afrontar las exigencias de un mundo moderno globalizado y en constante cambio, encontrando en la universidad un soporte social que les permita desarrollarse íntegramente; siendo esta tarea responsabilidad no sólo de autoridades, sino también de los propios estudiantes al fomentar el compañerismo y la solidaridad mediante el desarrollo de actividades que busquen este fin.

Recibido: 12-5-11 Aprobado: 20-6-11

Financiamiento: El estudio fue autofinanciado.

Conflictos de interés: El autor declara no tener conflictos de interés en la publicación de este artículo 
Tabla 5. Prevalencia de sintomatología depresiva en estudiantes de medicina peruanos.

\begin{tabular}{lcccccc}
\hline \multicolumn{1}{c}{ Autor } & Prevalencia & Ciudad & Universidad & Población (Año de estudios) & Instrumento & Año \\
\hline Pereyra-Elias R. et al (26) & $33,6 \%$ & Lima & Privada & 368 (1ro a 4to) & Escala de Zung 2010 \\
Galli-Silva E. et al (20) & $31,6 \%$ & Lima & Privada & 250 (4to, 5to, 6to) & MINI & 2001 \\
Osada J. et al (23) & $29,9 \%$ & Lima & Privada & 378 (1ro, 3ro, 5to, 7mo) & Escala de Goldberg & 2010 \\
Perales A. et al (8) & $29,6 \%$ & Lima & Publica & 1115 (1ro a 6to) & Escala de Zung & 2003 \\
Legua M, Arroyo H & $22 \%$ & Ica & Publica & 227 (1ro a 6to) & Escala de Zung 2007 \\
Castillo-Vilca MJ. et al (24) & $19 \%$ & Lima & Privada & 106 (5to) & CES-D & 2010 \\
Perales A. et al (21) & $19 \%$ & Trujillo & Publica & 645 (1ro a 6to) & Escala de Zung 2002 \\
Perales A. et al (8) & $18,7 \%$ & Trujillo & Publica & 508 (1ro a 6to) & Escala de Zung 2003 \\
\hline
\end{tabular}

\section{REFERENCIAS BIBLIOGRÁFICAS}

1. Brent DA, Birmaher B. Adolescent Depression. N Engl J Med 2002; 347:667-71.

2. Nair MK, Paul MK, John R. Prevalence of depresión among adolescents. Indian J Pediatr 2004; 71: 523-25.

3. Rondón M. Salud Mental: Un problema de salud pública en el Perú. Rev. Peru Med Exp Salud Pública 2006; 23(4): 237-38.

4. Organización Mundial de la Salud. Informe sobre la salud en el mundo 2001. Salud mental: nuevos conocimientos, nuevas esperanzas. [página de internet]. Fecha de acceso: Junio 2008. Disponible en: http://www.who.int/whr/2001/es/index.html

5. Instituto Especializado de Salud Mental "Honorio Delgado-Hideyo Noguchi”. Estudio epidemiológico metropolitano en salud mental 2002. Informe general. Lima: IESM HD-HN; 2002

6. Instituto Especializado de Salud Mental "Honorio Delgado-Hideyo Noguchi". Estudio epidemiológico en salud mental en la sierra peruana 2003. Lima: IESM HDHN; 2003.

7. Instituto Especializado de Salud Mental "Honorio Delgado-Hideyo Noguchi”. Estudio epidemiológico en salud mental en la selva peruana 2004. Lima: IESM HD-HN; 2004

8. Perales A, Sogi C, Morales R. Estudio comparativo de salud mental en estudiantes de medicina de dos universidades estatales peruanas. An Fac Med 2003; 64(4): 239-46.

9. Folse MI, Darosa DA, Folser R. The relationship between stress and attitudes toward leisure among first-year medical students. J Medical Educ 1985; 60 (8): 610-7.

10. Rosal MC, Ockene IS, Ockene JK, Barrett SV, Ma Y, Hebert JR. A longitudinal study of student's depression at one medical school. Academic Medicine 1997; 72(6):542-6.

11. Mosley TH, Perrin SG, Neral SM, Dubbert PM, Grothues CA, Pinto BM. Stress, coping and well-being among third-year medical students. Academic Medicine 1994; 69(9):765-7.

12. Lugo M, Lara C, González JE, Granadillo D. Depresión, ansiedad y estrés en estudiantes de medicina del área básica y clínica, su relación con el índice de lateralización hemisférica cerebral y el rendimiento académico. Universidad de Carabobo año 1999-2000. Arch venez de psiquiatr neurol 2004; 50(103): 21-29.

13. Cobb S. Social Support as a moderator of life-stress. Presidential Address Psychosom Med 1976; 38: 300.

14. Formoso D, Gonzales NA, Aiken LS. Family conflict and children's internalizing and externalizing behavior: Protective factors. Am J Community Psychol 2000; 28(2): 175-99.

15. Gaviria S, Rodríguez MDÁ, Álvarez T. Calidad de la relación familiar y depresión en estudiantes de medicina de Medellín, Colombia, 2000. Rev. chil. neuro-psiquiatr 2002; 40(1): 41-46.

16. Novara J, Sotillo C, Warthon D. Estandarización y correlación de las escalas de Beck, Hamiltoan y Zung para depresión en Lima Metropolitana. Lima, 1985.

17. Miranda-Bastidas CA, Gutiérrez-Segura JC, BernalBuitrago F, Escobar CA. Prevalencia de depresión en 
estudiantes de medicina de la U. del Valle. Rev. colomb psiquiatr 2000; 29(3):251-60.

18. Amézquita-Medina ME, González-Pérez RE, Zuluaga-Mejí D. Prevalencia de la depresión, ansiedad y comportamiento suicida en la población estudiantil de pregrado de la Universidad de Caldas, año 2000. Rev. colomb psiquiatr 2003; 32(4):341-56.

19. Czernik GE, Giménez S, Mora-Morel M, Almirón LM. Variables sociodemográficas y síntomas de depresión en estudiantes universitarios de Medicina de Corrientes, Argentina. Alcmeon Rev Arg Clín Neuropsiquiátr 2006;13(2):64-73.

20. Galli-Silva E, Feijoo-Llontop L, Roig-Rojas I, Romero-Elmore S. Aplicación del "MINI" como orientación diagnóstica psiquiátrica en estudiantes de medicina de la Universidad Peruana Cayetano Heredia. Informe preliminar epidemiológico. Rev Med Hered 2002; 13(1):19-25.

21. Perales A, Sogi C, Morales R. Salud mental de estudiantes de medicina de la universidad nacional de Trujillo. SITUA 2002; 11 (21): 14-20

22. Hojat M, Glaser K, Xu G, Veloski JJ, Christian EB. Gender comparisons of medical students' psychosocial profiles. Med Educ 1999; 33(5):342-9.
23. Osada J, Rojas M, Rosales C, Vega-Dienstmaier JM. Sintomatología ansiosa y depresiva en estudiantes de medicina. Rev Neuropsiquiatr 2010; 73(1):15-19

24. Castillo-Vilca MJ, Prado-Mendoza CJ, VegaDienstmaier JM. Prevalencia de depresión en estudiantes del quinto año de medicina de una universidad privada de Lima. Rev Neuropsiquiatr 2010; 73(1):9-14

25. Arriola-Quiroz I, Stucchi-Portocarrero S. Depresión en estudiantes de medicina: Una aproximación bibliométrica. Rev Med Chile 2010; 138:388-389.

26. Pereyra-Elías R, Ocampo-Mascaro J, Silva-Salazar V, Vélez-Segovia E, Costa-Bullón AD, Toro-Polo LM, Vicuña-Ortega J. Prevalencia y factores asociados con síntomas depresivos en estudiantes de ciencias de la salud de una universidad privada de Lima, Perú 2010. Rev Perú Med Exp Salud Publica 2010, 27(4):520-526.

Correspondencia: Dr. Hugo Arroyo Hernández Correo electrónico: hugoarroyo12@hotmail.com 